preceding 12 months in elderly with cardiovascular/cerebrovascular disease, or major cardiovascular risk factors. Vascular Depression isn't described in DSM-V, and that difficults clinical recognition and affects clinically informed systematic studies.

Objectives: Regarding a clinical case, we enphasize the clinical impact of Vascular Depression`s hypothesis.

Methods: We present a qualitative review of this topic using the Pubmed Central database.

Results: 74 years old male patient, with major depressive disorder about ten years. Depressive and cognitive symptoms didn't respond to antidepressive treatment and his functional state has gradually declined.

Conclusions: Vascular depression develops after the 60 - 65 years in the absence of personal and family history of affective disorder. The key symptoms are low energy, anhedonia, deficits in selfinitiation, psychomotor retardation, reduced processing speed and lack of insight into mood symptoms. Clinical assessment includes a review of history of vascular risk factors or/and disease, but also an imagiological evidence demonstrating subcortical white matter abnormalities. Insidious and chronic course tends to delay its recognition and management. This becomes critical because Vascular Depression is associated with poor response to antidepressant treatment and persistent depressive symptoms. It's also associated with poor selfmanagement of comorbidities and impairment in daily function. Increased mortality from all causes is widely documented.

Keywords: Vascular Depression; Geriatric depressive syndromes; Cardiovascular disease; Subcortical white matter abnormalities

\section{Oncology and psychiatry}

\section{EPP0866}

The impact of life hardiness, attachment style and personality profile on ptsd vulnerability manifestation in breast cancer patients undergone successful surgical treatment.

\author{
A. Vasileva ${ }^{*}$ and E. Lukoshkina \\ Non-psychotic Disorders Treatment And Psychotherapy, \\ V.M. Bekhterev National Research Medical Center for psychiatry and \\ neurology, saint petersburg, Russian Federation \\ ${ }^{\star}$ Corresponding author. \\ doi: 10.1192/j.eurpsy.2021.1147
}

Introduction: PTSD manifestation is determined by facing extreme life threatening experience going beyond our stress coping skills. The diagnosis of the serious illness like cancer or SARS-2 COVID 19 can be considered as one of the PTSD risk factors. In our clinical practice we have to distinguish the patients groups vulnerable to comorbid PTSD as well as define the psychological factors like good life hardiness, adaptive internal illness image or specific personality profile that can help to cope with disease stress and should be strengthen with psychosocial interventions.

Objectives: After screening with PTSD Trauma Screening Questionnaire and an expert clinical interview aimed to verify the PTSD diagnosis according to ICD-10 criteria 97 breast cancer patients were enrolled in the study, 46 with comorbid PTSD, 51 well coped with stress
Methods: Semi-structured interview, Hardiness Survey questionnaire,Experiences in Close Relationships-Revised (ECRR) Adult Attachment questionnaire,Impact of Event Scale-R - IES$\mathrm{R}$, the questionnaire of the internal disease model, Ego-structure test by G. Ammon(ISTA),

Results: The correlation analysis revealed negative correlation between PTSD diagnosis and hardiness, especially its Involvement, Control, Risk acceptance sub-scales and with the Traumatic event impact score. Deficient-destructive ISTA personality profile had a positive correlation with PTSD and traumatic Impact scores, the strongest correlation were with deficient aggression $(r=0,698$, $\mathrm{p}=0.01)$, destructive anxiety $(\mathrm{r}=0,674, \mathrm{p}=0,01)$, and deficient internal and external demarcation $(\mathrm{r}=0,678, \mathrm{p}=0,01)$. The adaptive internal illness image types had a negative correlation with PTSD Conclusions: Hardiness, maladaptive illness images types and destructive-deficient personality dimension should be the main targets for psychotherapy in comorbid PTSD treatment and prevention

Keyword: life hardiness ptsd breast cancer internal image of the illness construct anxiety social support

\section{EPP0867}

The role of self-compassion in the relationship between pain and depression in palliative patients

\author{
J. Guerra $^{1 \star}$, L. Santiago ${ }^{1}$ and D. Carreiras ${ }^{2}$ \\ ${ }^{1}$ Faculdade De Medicina Da Universidade De Coimbra, Universidade \\ de Coimbra, Coimbra, Portugal and ${ }^{2}$ Center For Research In \\ Neuropsychology And Cognitive And Behavioral Intervention, \\ University of Coimbra, Coimbra, Portugal \\ ${ }^{\star}$ Corresponding author. \\ doi: 10.1192/j.eurpsy.2021.1148
}

Introduction: In palliative care, depression and pain are prevalent variables with a reciprocal and controversial relationship. Depression is common in people with chronical diseases. In the last decades, self-compassion has been pointed as a protective psychological process to negative affect.

Objectives: The current study aimed to test the role of selfcompassion in the relationship between pain and depression in palliative patients.

Methods: Sample was composed of 33 patients in palliative care, with a mean of 74.12 years of age $(S D=12.76)$. Participants completed self-report questionnaires and data was analyzed using SPSS.

Results: From the descriptive analysis of the results of the Geriatric Depression Scale, 22 patients were depressed (66.6\%), 9 of them in severe depression (27.7\%). Self-compassion presented a negative and moderate correlation with depression. Depression was positively correlated with pain. A hierarchical regression to predict depression was conducted. Firstly, pain was entered as a predictive variable with a significant effect. Secondly, self-compassion was entered, and the model was significantly incremented, explaining $41 \%$ of depression. Only self-compassion was significant in this model.

Conclusions: Discussion and conclusion: The association between pain and depression in palliative care corroborate previous research. Results seemed to show that self-compassion has a significant effect in the relationship between pain and depression. Cultivating a compassion self-to-self relationship might have an 
important effect attenuating the link between pain and depression in palliative care.

Keywords: Pain; Depression; Palliative patients; Self-compassion

\section{EPP0868}

\section{The repercussions of burnout among health care} professionals in medical oncology in tunisia

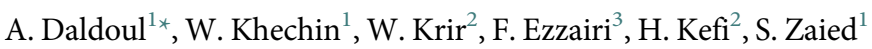
and S. Ben Ahmed ${ }^{3}$

${ }^{1}$ Medical Oncology, Fattouma Bourguiba University Hospital, Monastir, Tunisia; ${ }^{2}$ Psychiatry, Military Hospital, Tunis, Tunisia and ${ }^{3}$ Medical Oncology, University Hospital, sousse, Tunisia

${ }^{*}$ Corresponding author.

doi: 10.1192/j.eurpsy.2021.1149

Introduction: Freudenberger was the first to define burnout as a feeling of helplessness and guilt, as well as boredom and disinterest. Objectives: Our study aimed to analyze functional complaints and the behavior of healthcare professionals in this area associated with this syndrome.

Methods: This was a cross sectional study including health care professionals in medical oncology working in public hospitals in Tunisia. It was carried out from 15 January 2019 to 15 June 2019. Health professionals were asked to answer the Maslach -Burnout Inventory Test. Results: The average age was 34 years \pm 6.7 . Burn-out was found in 15 of the participants, (21\%). In our population, a high emotional exhaustion score was significantly associated with its repercussions: Sadness, Blockage, sleep disturbances, unexplained pain, Epigastralgia / fatigue, Addictive behavior ; avoidance behavior, repercussions on the relationship with those around them, desire for a transfer, regret for choosing a profession, suicidal thoughts, absenteeism and smoking. A high depersonalization score was significantly associated with several functional and behavior complaints, in particular: irritability, anger, feeling of indifference, guilt, unexplained pain, decreased performance, suicidal thoughts. A low personal achievement score was significantly associated with psychotropic drug use. Global burn-out was significantly associated with feeling of blockage, guilt, unexplained pain, epigastralgia and fatigue, addictive behaviors and avoidance behaviors as well as suicidal thoughts, absenteeism and consumption of psychotropic drugs.

Conclusions: Through its impact on professionals, burnout in medical oncology represents a major threat to the quality of care and the survival of institutions.

Keywords: burn out; oncology

\section{EPP0869}

\section{Burnout among health care professionals in medical oncology in tunisia}

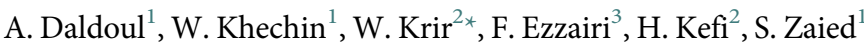
and S. Ben Ahmed ${ }^{3}$

${ }^{1}$ Medical Oncology, Fattouma Bourguiba University Hospital, Monastir, Tunisia; ${ }^{2}$ Psychiatry, Military Hospital, Tunis, Tunisia and ${ }^{3}$ Medical Oncology, University Hospital, sousse, Tunisia

${ }^{\star}$ Corresponding author.

doi: 10.1192/j.eurpsy.2021.1150
}

Introduction: Burnout syndrome concerns $27.8 \%$ of the general working population against $37 \%$ among doctors.

Objectives: This study aimed to report the prevalence of burnout among health care professionals in medical oncology in Tunisia.

Methods: This was a cross sectional study including health care professionals in medical oncology working in public hospitals in Tunisia. It was carried out from 15 January 2019 to 15 June 2019. Health profeessionals were asked to answer the Maslach -Burnout Inventory Test. Three scores allowing to locate the burn-out state of the person: the Score of emotional exhaustion (SEE), the Depersonalization score (SD) and the Score of personal achievement at work (SAP).

Results: Le taux de participation était de 58,3\%. La combinaison d'un SEE élevé, d'un SD élevé et d'un SAP bas définit le syndrome de Burn-out. Le SEE était élevé chez 44 travailleurs (63\%), indiquant un épuisement émotionnel sévère. Un SD élevé a été trouvé chez 37 répondants (53\%). La majorité des participants (59\%) avaient un PAS faible. Le burnout est défini par l'association chez la même personne d'un épuisement émotionnel élevé, d'une dépersonnalisation élevée et d'un faible rendement personnel. Cela a été trouvé chez 15 des participants (21\%). Selon l'étude analytique, le sexe féminin était significativement associé à un SEE élevé, un SAP bas et un épuisement global. Le travail de nuit était significativement associé à un degré élevé de dépersonnalisation et à un degré élevé d'épuisement général.

Conclusions: Burnout is linked to an increasingly ergonomic load. Health care professionnel, particularly in oncology, are frequently faced to this syndrome.

Keywords: burnout; oncology

\section{EPP0870}

\section{Burnout and predisposing factors in medical oncology} in tunisia

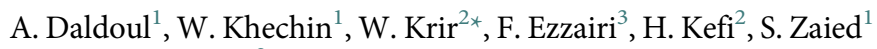 \\ and S. Ben Ahmed \\ ${ }^{1}$ Medical Oncology, Fattouma Bourguiba University Hospital, \\ Monastir, Tunisia; ${ }^{2}$ Psychiatry, Military Hospital, Tunis, Tunisia and \\ ${ }^{3}$ Medical Oncology, University Hospital, sousse, Tunisia \\ ${ }^{\star}$ Corresponding author. \\ doi: 10.1192/j.eurpsy.2021.1151
}

Introduction: Health care professionals are particularly concerned with burnout

Objectives: This study aimed to evaluate the the factors predisposing to occupational burnout

Methods: This was a cross sectional study including health care professionals in medical oncology working in public hospitals in Tunisia. It was carried out from 15 January 2019 to 15 June 2019. Health profeessionals were asked to answer the Maslach -Burnout Inventory Test.

Results: The mean age was $34 \pm 6.7$ years [23 - 57]. The sex ratio was 0.22 . Our study population included 37 doctors (53\%) and 33 nurses (47\%). The inappropriate working conditions mentioned by the participants were as follows: The requirementss of patients and their families $(91.5 \%)$, the lack of resources $(87 \%)$, overwork found $(83 \%)$, unsatisfactory effort- salary ratio $(83 \%)$ and the reduced number of staff (77\%). Several Burn-out factors mentioned by the participants were significantly associated with a high 